\title{
Handlungs- und Interaktionskrisen
}

\section{Eine Annäherung in systematisierender Absicht}

\author{
Alexander Antony • Gerd Sebald • Frank Adloff
}

(C) Springer Fachmedien Wiesbaden 2016

Zusammenfassung Der Beitrag führt in die Thematik des Sonderhefts ein und schlägt eine idealtypische Systematisierung von Handlungs- und Interaktionskrisen vor. Ausgehend von einer Arbeitsdefinition werden exemplarisch Ansätze aufgegriffen und skizziert, in denen krisenhaften Situationen eine theoriesystematisch relevante Bedeutung zukommt. Auf dieser Grundlage und der Zusammenschau der im Sonderheft versammelten Beiträge werden drei analytische Perspektiven auf Handlungs- und Interaktionskrisen ausbuchstabiert: die Akteur_innenperspektive, die ,distanzierte"Beobachter_innenperspektive und die Darstellungsperspektive. Abschließend wird dafür votiert, Handlungs- und Interaktionskrisen jenseits der dichotomen Unterscheidung von Mikro- und Makro-Phänomenen in den Blick zu nehmen.

Schlüsselwörter Handlungskrise · Interaktionskrise · Heuristik · soziologische Theorie $\cdot$ Sozialtheorie

\section{InterAction crises}

Toward a systematization

Abstract The article introduces the special issue's subject-matter and suggests a working definition and systematization of action and interaction crises. Based on

\footnotetext{
A. Antony $(\bowtie)$

Institut für Soziologie, Universität Wien, Rooseveltplatz 2, 1090 Wien, Österreich

E-Mail: alexander.antony@univie.ac.at

G. Sebald $(\bowtie)$

DFG-Graduiertenkolleg „Präsenz und implizites Wissen“, FAU Erlangen-Nürnberg,

Henkestraße 9-11, 91054 Erlangen, Deutschland

E-Mail: gerd.sebald@fau.de

F. Adloff $(\square)$

Institut für Soziologie, FAU Erlangen-Nürnberg, Kochstraße 4, 91054 Erlangen, Deutschland

E-Mail: frank.adloff@fau.de
} 
the volume's contributions as well as an outline of selected approaches to crises as theoretically relevant phenomena, it identifies three distinct analytical perspectives: that of the actor(s), that of the detached observer, and one that focuses on the crisis's representation. Any productive analysis of action and interaction crises, the authors argue, must move beyond the traditional micro-macro dichotomy.

Keywords Action crisis · Interaction crisis · Heuristics · Sociological theory · Social theory

\section{Von ,großen“" und ,kleinen“ Krisen in der Soziologie}

Krisen sind die Geburtshelfer der Soziologie. Die durch die Modernisierung verursachten Umbrüche im neunzehnten Jahrhundert - z. B. im ökonomischen und im politischen Feld, in der Medien- und Transporttechnik, in der fortschreitenden sozialen Differenzierung - ließen die eingelebten Muster vormals traditionaler Gesellschaften problematisch und ungültig werden. Damit wurde das Problem einer Erklärung dieser Transformationen und Disruptionen virulent. Antworten kamen, neben den Geisteswissenschaften, auch aus den sich entwickelnden Sozialwissenschaften, seien es Karl Marx' Analysen des Kapitalismus und seiner Krisen, Émile Durkheims Untersuchung der Anomien in zunehmend arbeitsteilig organisierten Gesellschaften oder Max Webers Analysen des sich verselbständigenden Geists des Kapitalismus. Es waren die großen Verwerfungen, Friktionen und Spaltungen, die von den Gründervätern der Soziologie untersucht und aus denen heraus die Grundlegungen der soziologischen Theorie und Methode entwickelt wurden.

Unbestritten ist, dass wir uns auch jetzt, mehr als hundert Jahre später, in einer Zeit teils weltumspannender Krisen bewegen. Allerdings wollen wir mit dem vorliegenden Sonderheft den soziologischen und nicht-soziologischen Krisendiagnosen und -erklärungen oder der Untersuchung der Auswirkungen von gesellschaftlichen Krisen kein weiteres Kapitel hinzufügen. Vielmehr ist der vorliegende Band von dem Grundgedanken inspiriert, dass es lohnen könnte, den soziologisch informierten Blick auf die „kleinen“, alltäglichen, aber oftmals nicht minder existenziellen Krisen zu richten. Denn obwohl die Soziologie durch diese zweifellos produktive theoretische sowie methodologische Impulse beziehen konnte, wurden sie kaum zum Gegenstand einer systematischen Beschäftigung.

Das vorliegende Sonderheft versteht sich insofern als ein Beitrag zur soziologischen Analyse und theoretischen Durchdringung dieser ,kleinen“ Krisen. Die enthaltenen Aufsätze behandeln - mit unterschiedlichen Zielsetzungen und aus verschiedenen theoretischen Perspektiven - Handlungs- und Interaktionskrisen. Sie blicken auf Prozesse der Entstehung von Krisen, auf deren Vermeidung, wie auch auf Versuche zu deren Bewältigung. Die untersuchten Phänomene reichen vom Umgang mit Demenzkranken bis hin zu Formen künstlerischer und politischer Praxis.

Wir möchten die Einleitung dazu nutzen, verschiedene Perspektivierungen und zentrale analytische Bestimmungselemente von Handlungs- und Interaktionskrisen, wie sie auch in unterschiedlichen Konstellationen in den einzelnen Beiträgen aufscheinen, in kondensierter Form herauszuarbeiten. Unsere Zielsetzung besteht dabei 
nicht primär darin, abstrakte theoretische Kategorien anzubieten. Vielmehr können die folgenden Ausführungen als ein Systematisierungsvorschlag verstanden werden, der darauf zielt, theoretische Heuristiken von Handlungs- und Interaktionskrisen, die ihre Funktion letztlich in der forschungspraktischen Arbeit finden, ein Stück weit voranzubringen.

\section{Von gesellschaftlichen Krisen zu Handlungs- und Interaktionskrisen - eine Arbeitsdefinition}

Der Begriff der Krisis bezeichnet im Griechischen eine Entscheidung oder entscheidende Wendung, sei es juristischer oder medizinischer Art. Bis in die Neuzeit wurde er vor allem in einem medizinischen Sinne gebraucht, seit dem 18. Jahrhundert fungierte er metaphorisch als ,Ausdruck einer neuen Zeiterfahrung, Faktor und Indikator eines epochalen Umbruchs“ (Koselleck 1982, S. 617). Krise bezeichnete damit eine zeitlich begrenzte Situation der Unsicherheit und Unentschiedenheit, die mit einer Entscheidung, einer Lösung beendet wird. In der soziologischen wie in der alltagssprachlichen Verwendung des Begriffes scheint sich die Konnotation aber tendenziell auf den Zeitraum vor der Lösung, auf die Situation der Unsicherheit, ja der Bedrohung zu verschieben. So bestimmt etwa Niklas Luhmann Krisen als ,unerwartete (thematisch nicht vorbereitete) Bedrohungen nicht nur einzelner Werte, sondern des Systembestands in seinem eingelebten Anspruchsniveau. Sie stimulieren und sammeln Aufmerksamkeit dadurch, dass sie den Erfüllungsstand zahlreicher Werte diffus, unbestimmt und unter Zeitdruck gefährden“ (2007 [1971], S. 16).

Krisen werden diesem Verständnis nach nicht im Hinblick auf krisenbewältigende Wendungen bestimmt, sondern es geraten insbesondere die Bedingungen ihres Auftretens in den Blick: Was jeweils als „Krise“ gelten kann, wird dementsprechend ex negativo vor dem Hintergrund eines theoretisch zu spezifizierenden Gegenbegriffs her gedacht. Dieser Theorielogik folgend, lassen sich Krisen nur im Hinblick auf nicht-krisenhafte Zustände konkretisieren. Gesellschaftliche Krisen sind demnach als Phänomene zu bestimmen, deren Auftreten dazu führt, dass ,ein etablierter, gesichert oder verlässlich erscheinender Sachverhalt fraglich oder instabil zu werden droht" (Friedrichs 2007, S. 14).

Für die Thematik des vorliegenden Sonderhefts ist nun die Beobachtung relevant, dass eine solche Argumentationsfigur, die analytisch auf die „Unordnung des Sozialen“ als kleinsten gemeinsamen Nenner zielt, sich keineswegs nur in gesellschaftstheoretischen Fassungen, d.h. etwa im Hinblick auf das Brüchig-Werden institutioneller oder systemischer Ordnungen, findet. Krisenhafte Phänomene haben stets auch das Interesse handlungs- und interaktionstheoretisch orientierter Soziolog_innen auf sich gezogen. So unterscheiden etwa Peter L. Berger und Thomas Luckmann ,zwei Möglichkeiten subjektiver Wirklichkeitsabsicherung [...]: Routine und die Bewältigung von Krisen“ (Berger und Luckmann 2007 [1969], S. 158). Es sind „Grenzsituationen“ (ebd., S. 159), wie der Tod, oder „Wirklichkeitskrisen“, die durch „Kontakte mit einer alternativen Wirklichkeit“ entstehen, welche die „routinemäßige [...] Wirklichkeitssicherung“ (ebd., S. 166) gefährden. Sie rufen entsprechende Bewältigungsstrategien (etwa Riten) und Gegenmaßnahmen auf den 
Plan. Erving Goffman hat, um ein zweites prominentes Beispiel zu nennen, in einer Vielzahl von ethnografischen Einzelstudien gezeigt, inwieweit situative Störungen (z. B. drohende Gesichtsverluste der Beteiligten) das Interaktionsgeschehen prägen, aber auch, wie derartige Interaktionsstörungen - oft auf subtile Art und Weise bewältigt werden oder ihr Manifestwerden - etwa durch taktvolles Ignorieren verhindert wird (Goffman 1967).

Auch wenn sich aus diesen Vorüberlegungen und Illustrationen freilich nicht umstandslos ein umfassendes Verständnis von Handlungs- und Interaktionskrisen ableiten lässt, so wollen wir doch auf der Grundlage des Gesagten eine vorläufige Arbeitsdefinition vorschlagen: Unter Handlungs- und Interaktionskrisen sind jene Situationen zu verstehen, die ein (zeitlich begrenztes) Versagen, eine situative Inadäquanz oder Unbrauchbarkeit von Handlungs- und Deutungsgewohnheiten im Hinblick auf die Gewährleistung des ,,normalen“ - und damit antizipierbaren Handlungs- bzw. Interaktionsverlaufs implizieren. Diese Minimaldefinition soll die folgenden Überlegungen anleiten, aber auch im Hinblick auf ihre analytische Tiefenschärfe hinterfragt und nach und nach konkretisiert werden. Informiert durch sie lässt sich in einem nächsten Schritt der Blick exemplarisch auf sozial- und handlungstheoretische Ansätze richten, in denen Handlungs- und Interaktionskrisen eine theoriesystematisch relevante Bedeutung zukommt.

\section{Fragmente einer Theorie der Handlungs- und Interaktionskrisen}

Im Pragmatismus, im phänomenologisch orientierten Ansatz von Alfred Schütz, der Ethnomethodologie Harold Garfinkels oder der objektiven Hermeneutik Ulrich Oevermanns wird dem Konzept der Krise bzw. dem Auftreten problematischer Situationen ein besonderer Stellenwert zugewiesen. Diesen Versuchen einer theoretischen Fassung der Genese, des Ablaufs und der Bewältigung von Handlungsproblemen kommt auch für die Konzeptualisierung von Handlungs- und Interaktionskrisen eine entscheidende Rolle zu.

Pragmatisch orientierte Sozialtheorien (Mead, Dewey, Heidegger, Wittgenstein) richten ihr Augenmerk auf die intersubjektiv geteilte Praxis von Lebensformen, auf den pragmatischen Vollzugscharakter von Handlungskoordination und Interaktion. Dabei rekurrieren die genannten Autoren immer wieder auf die fundamentale Rolle körperlicher, sensomotorischer und präreflexiver Dispositionen und Gewohnheiten (vgl. Dewey 1983 [1922]). Kognitive bzw. reflexive Formen der Handlungskoordination bleiben in diesen Theorieansätzen insofern theoretisch sekundär, als sie aus der praktischen Tätigkeit abgeleitet werden.

Im Hinblick auf Handlungs- und Interaktionskrisen ist dabei interessant, dass das Problematischwerden des primären Weltbezugs, d. h. eine wie auch immer geartete „Störung“ habitueller Handlungskoordination, als Bedingung der Möglichkeit des Auftretens reflexiver Operationen fungiert: Dewey und Mead bestimmen auf Symbolverwendung beruhende mentale Phasen des Handelns als funktional im Hinblick auf die Lösung problematischer Situationen. D. h., reflexives Handeln hat seinen Ursprung in der situativen Inadäquanz gewohnheitsmäßigen Handelns und dient der Bewältigung von Handlungsproblemen. Derartige „,verzögerte Reaktionen“ (Mead 
1973 [1934], S. 137 ff.), die sich durch eine symbolisch prozessierende Imagination alternativer Handlungsmöglichkeiten auszeichnen, lassen sich von routinierten „unmittelbaren Reaktion[en]“ unterscheiden (ebd.; vgl. auch Dewey 1998 [1929], S. 152 ff., 223). Krisenhafte Situationen sind aus dieser Sicht konstitutiv für die Entstehung eines reflexiven Bewusstseins und auf Symbolverwendung basierender Wissenspraktiken, die sich durch eine (partielle) Abstraktion vom nicht-reflexiven Erfahren im Hier-und-Jetzt auszeichnen. Das pragmatistische Handlungsmodell lässt sich insofern (zumindest in groben Zügen) als Abfolge von Gewohnheit - Störung/ Krise - Reflexion - Gewohnheit rekonstruieren und bietet damit einen basalen handlungstheoretischen Ausgangspunkt für die Konzeptualisierung von Handlungs- und Interaktionskrisen (vgl. auch Oechsle und Reimer in diesem Band).

Ähnlich wie der Pragmatismus geht auch die pragmatische Lebenswelttheorie Schütz'scher Prägung von einer als selbstverständlich gegebenen Welt des alltäglichen Lebens aus. Bestimmte Aspekte dieser Welt sind durch subjektive oder auferlegte Selektionsleistungen als ,problematische Möglichkeiten“ hervorgehoben und werden im Modus des Handelns bearbeitet. Problematisch sind dabei für Schütz (mit Rückgriff auf Husserl) Möglichkeiten, die in Widerstreit bzw. Konkurrenz zueinander stehen (vgl. Schütz 2004 [1951], S. 267 ff.). Ein bestimmter Teil des vorund zuhandenen intersubjektiven Wissensvorrats wird zweifelhaft, seine Gewissheit und Geltung schwinden und müssen wieder hergestellt werden. In die Krise geraten demnach die Typisierungen und Generalisierungen: „Die auf der Konstanz der Weltstruktur aufgebauten Erwartungen mögen ,explodieren ‘, das Gültige zweifelhaft, das Vermögliche undurchführbar werden. Das vordem als fraglos-gegeben Angesetzte wird dann zum Problem, einem theoretischen, praktischen oder emotionalen, das formuliert, analysiert und gelöst werden muss“ (Schütz 2003 [1957], S. 327, vgl. auch Sebald 2014, S. 80 f.). Wenn diese „Explosionen“ unsere Wissensstruktur, unsere „relativ-natürliche Weltanschauung“, wie Schütz in Anlehnung an Scheler formuliert, als Ganzes und grundlegend in Frage stellen, dann spricht er von „Krise“. Darunter versteht Schütz ,alle Arten von religiösen, ehelichen, geschäftlichen, gesundheitlichen Schwierigkeiten im Einzelleben oder von Naturkatastrophen, Krieg, Zusammenbrüchen des ökonomischen oder sozialen Systems im Leben der Gruppe“ (Schütz 2003 [1957], S. 343). Das in die Krise geratene Wissen bringt die bisherige „Weltanschauung“ ins Wanken. Mit der Auflösung der Typisierungen und Erwartungen entsteht potenziell auch Neues, das vorher durch die gültigen Wissenselemente blockiert war.

Mit der Ethnomethodologie Harold Garfinkels liegt eine an Schütz anschließende Position vor (vgl. Eberle 2008), die moralische bzw. soziale Ordnung als fortlaufend durch die ,rule governed activities of everyday life“ generiert begreift: „A society’s members encounter and know the moral order as perceivedly normal course of action - familiar scenes of everyday affairs, the world of daily life known in common with others and with others taken for granted" (Garfinkel 1967, S. 35). Die Ethnomethodologie geht von der Grundannahme aus, dass Regeln, Hintergrunderwartungen (background expectancies) und andere strukturelle Voraussetzungen alltäglicher Interaktionen in gelingenden bzw. routinehaften Interaktionssituationen latent (seen but unnoticed) bleiben. Sie stellen nach Garfinkel die ,routine grounds of everyday activity“ (ebd.) dar. Um die stillschweigenden Selbstverständlichkeiten alltäglicher 
Handlungsvollzüge sichtbar zu machen, setzt die Ethnomethodologie - im Gegensatz zu den bereits genannten Ansätzen weniger theoretisch denn methodologisch orientiert - auf fingierte Störungen ,normaler“ Abläufe, die berühmten breaching experiments (vgl. dazu ausführlich Krämer in diesem Band). Insbesondere Interaktionskrisen sind für diesen Ansatz vor allem ein methodisches Mittel, um soziologische Erkenntnis zu generieren: Die kontrolliert erzeugte Unordnung gewährt einen Blick auf die im Alltagshandeln zumeist unhinterfragten und nicht problematisierungsbedürftigen praktischen Methoden der situativen Herstellung von sozialer Ordnung.

Schließlich liegt mit dem strukturtheoretischen Ansatz Ulrich Oevermanns eine Position vor, die Krisen, und ihren Komplementärbegriff Routine, als konstitutive Elemente des Sozialen überhaupt in den Blick nimmt (vgl. ausführlicher Garz und Raven 2015, S. 25-59). Schon die menschliche Erfahrung ist nach Oevermann grundlegend krisenhaft, weil sie sich einer prädikativen Bestimmung und damit einem routinehaften Vollzug immer wieder entzieht. Die „Dialektik von Krise und Routine“ (Oevermann 2006, S. 95) ist in den menschlichen Erkenntnismöglichkeiten selbst angelegt als ,ein dem Gebrauch von Sprache innewohnendes Grundverhältnis“ (Oevermann 2008, S. 9). Das Auftreten von Krisen ist demnach abhängig von der anthropologischen Ausstattung des Menschen, dem vorhandenen sprachlich codierten Wissensvorrat sowie der kulturellen ebenso wie der individuellen Entwicklung (ebd., S. 11). Der daraus abgeleitete „Begriff der Lebenspraxis als Zentrum der Krisenfähigkeit und der Krisenbewältigung“ (ebd., S. 18) dient Oevermann im Weiteren zu einer Typologisierung der Krisen in traumatische, ästhetische und Entscheidungskrisen. Krisen stellen in diesem strukturalistischen Ansatz vor allem einen Mechanismus dar, der einerseits die Entstehung von Neuem erklären kann, insofern alles Wissen als „Ergebnis einer Krisenlösung“ (Oevermann 2006, S. 97) interpretiert wird: Wissen ,gehört also zur Sphäre von Routinen, die sich entweder schon bewährt haben oder deren Bewährung aussichtsreich erwartet wird“ (ebd.). Andererseits fragt Oevermann nach den gesellschaftlichen „Orten“ und Weisen der Krisenbewältigung (etwa im professionalisierten Handeln) und eröffnet damit ein weites soziologisches Feld.

Ersichtlich wird, dass die exemplarisch besprochenen Ansätze ein starkes Interesse an problematischen Situationen bzw. Handlungs- und Interaktionskrisen erkennen lassen. In den Blick gerät die Frage nach einer handlungstheoretischen Fassung des Nicht-Krisenhaften als notwendigem Komplementärbegriff zum Krisenhaften. In systematischer Hinsicht relevant ist einerseits, dass die jeweiligen ins Treffen geführten Konzepte des Nicht-Krisenhaften (Gewohnheiten, Typisierungen, Hintergrunderwartungen, Routinen) allesamt auf die Wissensdimension sozialen Handelns abstellen und Wissen andererseits, sei es nun verkörpert und implizit oder propositional ,verfasst“, als sozial geteilt und distribuiert charakterisiert wird. Darüber hinaus weisen die Ansätze unterschiedliche Möglichkeiten aus, Krisenbewältigungsstrategien handlungstheoretisch zu fassen - etwa im Hinblick auf die Funktionalität symbolischer Abstraktionsleistungen - und beschreiben Krisensituationen als „Generatoren" der Entstehung von Neuem, das sich z. B. in modifizierten Gewohnheiten, neu etablierten Routinen oder Deutungen manifestiert. Festhalten lässt sich allerdings auch, dass, obwohl die behandelten Ansätze zweifelsohne einen systematisch 
relevanten Beitrag zu einer theoretischen Konzeptualisierung von Handlungs- und Interaktionskrisen leisten können, sie nichtsdestoweniger - sieht man von Oevermann ab - keinen verbindlichen Krisenbegriff explizieren bzw. Krisen (mitunter) nicht systematisch von Handlungsproblemen im Allgemeinen unterscheiden.

\section{Drei Perspektiven auf Handlungs- und Interaktionskrisen}

Dies führt unmittelbar zur Notwendigkeit, Handlungs- und Interaktionskrisen insofern weiter zu spezifizieren, als nicht jede „Bagatellstörung“ (Bergmann 2013, S. 286), nicht jedes Handlungsproblem sinnvollerweise als „Krise“ zu definieren ist. Der Blick ist dementsprechend auf ,größere Rupturen des Sozialen“ (ebd.) auf der Handlungs- und Interaktionsebene zu richten. Soziologische Begriffe von Handlungs- und Interaktionskrisen drohen ansonsten ihr heuristisches Potenzial einzubüßen und zu ,all inclusive“-Konzepten zu verkommen. In dieser Hinsicht ist Martin Endreß und Nicole Zillien zuzustimmen, dass ,Vorbehalte[n] gegen die analytische Trennschärfe der Krisenbegrifflichkeit“ - und dies gilt sowohl für gesellschaftliche als auch für Handlungs- und Interaktionskrisen - nur dann beizukommen ist, „wenn die Disziplin erstens die als krisenhaft bezeichneten Situationen empirisch ausleuchtet, zweitens in komparativer Perspektive mögliche Besonderheiten aktueller Szenarien herausarbeitet und [...] drittens die Frage nach Angemessenheit und Deutungspotential der Krisensemantik stellt“" (2013, S. 416).

Die Beiträge des vorliegenden Sonderhefts kommen anhand der Untersuchung spezifischer Gegenstandsbereiche der Aufforderung nach einer empirischen Ausleuchtung und/oder einer konzeptuellen Analyse nach. In ihrer Zusammenschau und im Vergleich der verwendeten Krisenkonzepte sowie unter Heranziehung des oben erarbeiteten theoretischen Überblicks bieten sie die Möglichkeit, drei unterschiedliche Perspektiven auf Handlungs- und Interaktionskrisen auszubuchstabieren. Diese sind als idealtypische Möglichkeiten zu verstehen, Handlungs- und Interaktionskrisen konzeptuell zu fassen. Die eine, ,richtige“ Lösung kann es schon allein deswegen nicht geben, weil es sich bei Konzepten von Handlungs- und Interaktionskrisen um allgemeintheoretische Heuristiken, um sensitizing concepts (vgl. Blumer 1986 [1969], S. 147 f.) handelt, die den Forschungs- und Analyseprozess zwar anleiten und die gegebenenfalls auch empirisch irritierbar, aber eben nicht falsifizierbar sind. Im empirischen Forschungsprozess, das zeigt sich auch in den Beiträgen, sind vielfältige Überschneidungen als auch parallele Verwendungsweisen unterschiedlicher Krisenbegriffe denkbar.

\subsection{Die Akteur_innenperspektive: Krisen als Sinnzusammenbrüche bzw. als Erfahrungen der Unbewältigbarkeit}

Die erste Perspektivierung, die Krisen - je nach theoretischer Tradition, an die angeschlossen wird - als Situationen des Sinnzusammenbruchs bzw. als Erfahrungen der Unbewältigbarkeit auffasst, setzt analytisch konsequent beim Standpunkt bzw. der Perspektive der Handelnden an. Handlungsprobleme bzw. problematische Situationen stellen theoretisch die Bedingung der Möglichkeit von Sinnzusammenbrüchen 
bzw. Erfahrungen der Unbewältigbarkeit dar. Da aber nicht jedes Handlungsproblem per se krisenhaft sein kann, stellt sich die Frage, was problematische Situationen für die Handelnden zu krisenhaften macht. In der Tradition des Pragmatismus, der phänomenologisch orientierten Soziologie Schütz', aber auch in der Ethnomethodologie kann davon ausgegangen werden, dass Bedeutung bzw. Sinn Situationen im Allgemeinen und problematischen Situationen im Besonderen nicht gleichsam inhärent ist, sondern sich erst - ,strikt korrelativ und gleichzeitig“ (Dewey 2003 [1896], S. 244) - durch die „Reaktionen“ bzw. Haltungen (Mead) oder Deutungen (Schütz) der Handelnden konstituiert. ${ }^{1}$ D. h., problematische Situationen werden dann zu Krisen, wenn sich Handelnde in spezifischer Art und Weise gegenüber diesen Situationen verhalten. ${ }^{2}$

Will man zu einem theoretisch gehaltvollen Begriff von Handlungs- und Interaktionskrisen vorstoßen, dann kann das entsprechende problemauslösende Ereignis nicht für sich - isoliert - in den Blick genommen werden. Es besteht die Notwendigkeit, in der Reaktion der Handelnden auf problematische Sachverhalte (die streng genommen erst durch die Reaktion zu solchen werden) nach einem theoretischen Bestimmungsgrund des Krisenhaften zu fahnden.

Bei Krisen aus der Akteursperspektive handelt es sich also nicht um im Alltag gleichsam omnipräsent vorkommende Situationen, die, obwohl sie z. B. Routinebrüche darstellen, nach einer gewissen Zeit für die Handelnden bewältig-, auslegoder normalisierbar sind (vgl. beispielhaft Schubert und Rammert 2006 und Meyer in diesem Band). Krisen liegen, so unser Vorschlag, vielmehr dann vor, wenn Handlungs- und Deutungsmöglichkeiten bzw. Normalisierungsstrategien (zumindest temporär) nicht mehr greifen. Man ist gewissermaßen mit seinem Latein am Ende, das habituelle Handeln (Dewey, Mead), die natürliche Einstellung (Schütz) oder die background expectancies (Garfinkel) sind in einem Ausmaß problematisch geworden, dass sozial approbierte Handlungsanschlüsse und Deutungsversuche schlicht nicht mehr möglich sind. Aus der Perspektive der Handelnden manifestiert sich eine derartige Situation in einer Erfahrung der Unbewältigbarkeit bzw. eines Sinnzusammenbruchs.

Die Krise bzw. das Krisenhafte stellt nach dieser ersten Perspektivierung demnach eine spezifische Qualität des bedeutungsvollen Erfahrens bzw. Erlebens von Handlungs- oder Interaktionssituationen dar, deren Bedeutungshaftigkeit sich paradoxerweise in ihrer senselessness (vgl. Garfinkel 1967, S. 54 f.) manifestiert. Anders ausgedrückt werden problematische Situationen zu Krisen, wenn die Antwort, die betroffene Handelnde darauf zu geben im Stande sind, sich in einer praktischen Nicht-Beantwortbarkeit manifestiert: Man weiß oder kann nicht mehr weiter. Eine derartige krisenhafte Qualität bezieht ihre subjektive Evidenz und Unausweichlichkeit (gegebenenfalls auch Ausweglosigkeit), wie Garfinkel anschaulich gezeigt hat,

\footnotetext{
${ }^{1}$ Die Betonung dieses ko-konstitutiven Verhältnisses zwischen problematischer Situation und Verhalten bzw. Deutung schließt die Zurkenntnisnahme der Sequenzialität von Krisenentstehungsprozessen keineswegs aus. In manchen Fällen mag der Sinnzusammenbruch sofort eintreten, in anderen erst nach einer Reihe von Normalisierungs-, Deutungs- oder praktischen Bewältigungsversuchen.

2 Ähnlich Bergmann im Hinblick auf Interaktionsstörungen: „Nicht das ,störende Ereignis“ ist schon die Unterbrechung [der Interaktion; die Hg.], sondern erst die Unterbrechung macht aus einem konkurrierenden Ereignis eine Störung“" (2013, S. 288).
} 
oftmals durch eine starke Affiziertheit der Handelnden - wie etwa Ärger, Angst oder, man denke an Helmuth Plessner (2003 [1941], S. 275 f.), Schwindel, Lachen und Weinen. ${ }^{3}$ Man wird gewissermaßen auf sich und seine Unzulänglichkeit, die problematische Situation praktisch zu bewältigen oder sinnhaft auszulegen, zurückgeworfen. ${ }^{4}$

\subsection{Die ,distanzierte“ Beobachter_innenperspektive: Krisen als Abweichungen}

Bei der zweiten Perspektivierung handelt es sich um eine Krisenkonzeption, die nicht „direkt“ beim Standpunkt der Handelnden, deren Erfahrungen etc. ansetzt, sondern Handlungs- und Interaktionskrisen aus einer „distanzierten“ soziologischen Beobachter_innenperspektive heraus bestimmt. Dabei besteht die Notwendigkeit der Angabe (oder der Unterstellung) einer „Normalfolie“ - d. h. Vorstellungen darüber, wie spezifische Handlungen und Interaktionen ,normalerweise“ ablaufen oder abzulaufen haben - und eines empirisch zu erbringenden Nachweises, in welcher Art und Weise bzw. gegebenenfalls auch in welchem Ausmaß von dieser Normalfolie abgewichen wird. Die Antwort auf die Frage danach, was als signifikante Abweichung gelten kann und damit die Verwendung des „Krisen“-Etiketts rechtfertigt, ist von den soziologischen Beobachter_innen zu erbringen und in einem doppelten Sinne relativ - relativ zur verwendeten Normalfolie und relativ zu den in Anschlag gebrachten Kriterien, anhand derer darüber entschieden wird, unter welchen Bedingungen eine krisenhafte und nicht bloß eine ,normale“ bzw. ,im Rahmen“ bleibende Abweichung vorliegt.

Derartige Normalfolien mögen - je nach Fall - mehr oder weniger stark konkretisiert werden und sie variieren hinsichtlich ihrer Spezifität. So können sie sich etwa auf alltagsweltliche Interaktionen im Allgemeinen beziehen, wonach Krisen sich folgerichtig durch eine Außeralltäglichkeit auszeichnen würden (vgl. etwa Brichzin in diesem Band). Sie können aber z. B. auch Handlungsverläufe und Interaktionsprozesse in bestimmten sozialen Feldern, institutionellen Settings, Sub-Kulturen, Szenen oder etwa spezifische Interaktionsformate (wie journalistische Interviews

\footnotetext{
3 Aufschlussreich sind in diesem Zusammenhang auch die von Garfinkel angegebenen - im Falle der breaching experiments idealerweise herzustellenden - Bedingungen, die das Auftreten derartiger Sinnzusammenbrüche begünstigen: ,(a) making it difficult for the person to interpret his situation as a game, an experiment, a deception, a play, i.e., as something other than the one known according to the attitude of daily life as a matter of enforcable morality and action [Uneinordenbarkeit; die Hg.], (b) making it necessary that he reconstruct the ,natural facts ' but giving him insufficient time to manage the reconstruction with respect to required mastery of practical circumstances for which he must call upon his knowledge of the ,natural facts, ' [Zeitmangel; die Hg.] and (c) requiring that he manage the reconstruction of the natural facts by himself and without consensual validation“ [mangelnde soziale Anerkennung; die Hg.] (1967, S. 54). Zum letzten Aspekt vgl. auch Berger und Luckmann 2007 [1969], S. 160 ff.

4 Insofern könnte man Krisensituationen im Anschluss an Meads Definition von Subjektivität, die er auf das Auftreten problematischer Situationen bezieht (vgl. 1987 [1903], S. 122 ff.), als ,Situationen gesteigerter Subjektivität“ charakterisieren. Vgl. auch Oevermann 2006, S. $111 \mathrm{f}$.
} 
oder politische Debatten) zum Gegenstand haben. ${ }^{5}$ Ohne an dieser Stelle Anspruch auf Vollständigkeit erheben zu wollen, lassen sich beispielhaft Normalfolien benennen, die in den Beiträgen dieses Bandes Verwendung finden:

- Idealtypische, nicht notwendig im engeren Sinne empirisch fundierte Vorstellungen darüber, wie funktionierende Handlungs- und Interaktionssituationen - etwa im Hinblick auf interaktionstheoretische Grundannahmen (vgl. Radvanszky in diesem Band) oder Konventionen der Redeübergabe (vgl. Schmidtke in diesem Band) - sich ,normalerweise“ ausgestalten.

- Das common sense knowledge der Forschenden, das es, als Kontrastfolie fungierend, erlaubt, abweichende Fälle zu identifizieren (vgl. Brichzin in diesem Band).

- Empirische Studien über gelingende Interaktionen als Vergleichsmaßstab, auf dessen Grundlage nicht gelingende, krisenhafte bestimmt werden können (vgl. Meyer in diesem Band).

- Handlungen und Zuschreibungen der an Krisensituationen Beteiligten, die z. B. ihren Unmut zum Ausdruck bringen und derart auf der Grundlage wirksamer Relevanzsysteme über Abweichungen „Auskunft“" geben.

\subsection{Die Darstellungsperspektive: Krisen als Teilnehmer_innen-Konstruktionen}

Der zuletzt genannte Punkt stellt bereits einen Grenzfall zur dritten Perspektivierung dar. Diese begreift Handlungs- und Interaktionskrisen als Teilnehmer_innenKonstruktionen. Entsprechend setzt man zwar bei den Akteursperspektiven an, doch Handlungs- und Interaktionskrisen werden hier, im Unterschied zum ersten Definitionsvorschlag, nicht per se in der Erfahrung der Unbewältigbarkeit bzw. des Sinnzusammenbruchs identifiziert - ein gleichsam ,absolutes“ Kriterium. Es wird vielmehr danach fragt, auf welche Art und Weise Handelnde Krisensituationen als solche für Mithandelnde anzeigen und kommunizieren bzw. wie diese sich in ihrem Tun dokumentieren. Mit Garfinkel ließe sich fragen: Wie werden Handlungs- und Interaktionskrisen durch die Teilnehmer_innen accountable und damit ,observableand-reportable“ (1967, S. 1) gemacht?

Ein derartiger Zugriff stellt dementsprechend nicht nur auf die von den Handelnden verwendeten Semantiken, Kategorien etc. ab, sondern auch auf die Art und Weise, wie sich Krisenkonstruktions-, aber auch Krisenbewältigungsprozesse sequenziell und interaktiv im Detail vollziehen, sowie auf die Frage, welche (gegebenenfalls impliziten) Sozio-Logiken derartigen Prozessen zugrunde liegen (vgl.

\footnotetext{
5 Damit ist freilich impliziert, dass das, was in manchen Handlungsbereichen als „,normal“, ,rational“, „schön“ usw. etikettiert wird, in anderen keineswegs als solches gelten muss. Während etwa die MusikForm „Noise“, der sich Kai Ginkel in diesem Band widmet, Uneingeweihte vor erhebliche Deutungsprobleme stellt und das Gehörte gegebenenfalls als „Krach“ etikettieren lässt (eine Normalisierungsstrategie), sehen Angehörige der Community darin eine künstlerisch wertvolle Praxis.
} 
Pfadenhauer und Dukat; Kissmann; Schröder in diesem Band). ${ }^{6}$ Der wohl eindeutigste (vielleicht auch simpelste) Fall eines solchen Krisenkonstruktionsprozesses ist in der Verwendung der Krisensemantik durch beteiligte Handelnde zu identifizieren - d.h., wenn spezifische Ereignisse explizit als „Krise“ bezeichnet werden. Allerdings ist davon auszugehen, dass ein derartiger Zugriff allein der Heterogenität von Krisenkonstruktionsprozessen nicht gerecht werden würde.

Dementsprechend besteht unser Vorschlag darin, Handlungs- und Interaktionskrisen als Teilnehmer_innenkonstruktionen auf einem Kontinuum zwischen der expliziten Verwendung der Krisensemantik einerseits und andererseits jenen Fällen zu situieren, in denen sich krisenhafte Situationen auf vielfältige andere Art und Weise dokumentieren. Je weiter weg man sich freilich von expliziten sprachlichen Krisenzuschreibungen bewegt, desto unbestimmter wird auch der Bezugsrahmen und damit umso „offener“ die Möglichkeiten der soziologischen Beobachter_innen, Handlungs- und Interaktionskrisen zu identifizieren. Das heißt, das Problem, das bereits bei der zweiten Krisenheuristik ausgemacht wurde - die Relativität und Kontingenz der Bestimmung von Handlungs- und Interaktionskrisen - besteht auch bei der dritten Perspektivierung: Letztlich bleibt es der/m soziologischen Beobachter_in überlassen, zu bestimmen, ob eine Krisensituation vorliegt oder nicht. Die Herausforderung besteht darin, nicht der Beliebigkeit Vorschub zu leisten, sondern plausibel und nachvollziehbar zu machen, auf der Grundlage welcher Kriterien man operiert. Unabhängig von konkreten Gegenstandsbereichen und entsprechenden Methodenrepertoires und Analysestrategien lässt sich aber theoretisch nicht vorschreiben, was als praktikabelster Weg anzusehen ist.

Wir wollen an dieser Stelle lediglich darauf hinweisen, dass interessanterweise gerade die forschungspraktische Kombination der unterschiedlichen vorgeschlagenen Perspektivierungen eine Möglichkeit bietet, mit derartigen Unbestimmtheitsproblemen umzugehen. Um dies am Beispiel der Verschränkung der ersten mit der dritten Krisenheuristik festzumachen: Man könnte etwa, mit der Darstellungsperspektive operierend, unter Hinzuziehung der ersten Krisendefinition, die ein „starkes“ Kriterium für die Identifikation von Handlungs- und Interaktionskrisen ins Treffen führt (Sinnzusammenbruch, Erfahrung der Unbewältigbarkeit), solche Situationen als Krisen beschreiben, die von den beteiligten Handelnden selbst, als unbewältigbar markiert bzw. kommuniziert werden. Eine derartige Perspektive erlaubt es z. B., Interaktionen, die von den Beteiligten als nahe dem Abbruch stehend angezeigt oder tatsächlich abgebrochen werden, als Interaktionskrisen zu charakterisieren: Die interaktive Bewältigung der Situation gelingt nicht oder wird zumindest von den Handelnden in Frage gestellt (vgl. Schmidtke in diesem Band). Dass sich ein solcher analytisch-kombinatorischer Zugriff als heuristisch produktiv erweisen könnte, zeigt sich auch darin, dass ganze Berufszweige (wie z. B. die Ärzteschaft, Psychotherapie, Sozialarbeit etc.) auf die stellvertretende Bewältigung individuell unbewältigbarer

\footnotetext{
6 Theoretisch a priori gesetzt ist bei einer derartigen Perspektive überdies nicht, dass es sich dabei um im engeren Sinne intendierte Prozesse bzw. absichtsvolles Tun handeln muss. Gerade wenn man in Rechnung stellt, dass sich Handlungs- und Interaktionskrisen oftmals auch affektiv-leiblich ,dokumentieren“ (vgl. etwa Kissmann; Krämer; Schmidtke in diesem Band) ist vielmehr an eine fungierende Intentionalität zu denken (vgl. Merleau-Ponty 1966 [1945], S. 474 ff.).
} 
Handlungsprobleme spezialisiert sind. Dabei fungiert das Eingeständnis einer derartigen Unbewältigbarkeit durch die Betroffenen oftmals auch als praktische Voraussetzung für die Inanspruchnahme professioneller Hilfeleistung. Professionalisierte Expert_innen erweisen sich in dieser Hinsicht nicht nur als assistierendes „Personal“ bei der Krisenbewältigung, sondern auch als Krisenkonstruktionspersonal (vgl. Pfadenhauer und Dukat; Schröder in diesem Band).

Gerade in der Kombination unterschiedlicher Perspektivierungen zeigt sich nicht nur, dass sich diese gegenseitig forschungspraktisch informieren können, sondern auch, dass die idealtypischen Definitionen als Reflexionsinstrumente dienen können, wenn es darum geht, unterschiedliche Probleme bei der Identifikation von Handlungs- und Interaktionskrisen zu explizieren und diese entsprechend forschungspraktisch zu bewältigen.

\section{Jenseits von „Mikro“ und „Makro“}

Abschließend bleibt festzuhalten, dass die hier vorgeschlagene Fokussierung auf Handlungs- und Interaktionskrisen als eigenständiges Thema der Soziologie keineswegs eine dichotome Gegenüberstellung von „Mikro“- und „Makro“-Phänomenen impliziert. ${ }^{7}$ Was vielmehr in allen Beiträgen dieses Bandes deutlich wird, ist, dass auch (und gerade) beim Auftreten von Handlungs- und Interaktionskrisen unterschiedliche „Ebenen des Sozialen“, unterschiedliche Kontexte und ausdifferenzierte Ordnungsbereiche wirksam werden (vgl. Giddens 1984, S. 61, Oechsle und Reimer sowie Schatzki in diesem Band). Sie fungieren sowohl als unmittelbare oder intervenierende Bedingungen des Auftretens als auch des Bewältigens von krisenhaften Situationen. Das gilt für Aspekte, die gemeinhin eher dem Mikro-Bereich zugerechnet werden, wie z. B. körperliche Routinen, auftretende Emotionen, das subjektive Bewusstsein oder das material-gegenständliche Umfeld, ebenso wie für semantische Ordnungsstrukturen (etwa Leitbilder oder Diskurse), für Institutionen oder Organisationen sowie für verschiedene gesellschaftliche Funktionsbereiche (etwa die Politik, die Kunst oder die für Dysfunktionalitäten besonders anfällige Ökonomie). Gerade im Auftreten von Handlungs- und Interaktionskrisen, so könnte aus diesem Befund abgeleitet werden, zeigen sich - je nach Fall - die Wirksamkeit, der Antagonismus oder das Zusammenwirken von gesellschaftlichen Strukturierungen in der alltäglichen Lebenswelt. Von daher ist die Annahme zurückzuweisen, dass der Blick auf Handlungs- und Interaktionskrisen lediglich einen Mikrokosmos des Sozialen vor-

\footnotetext{
7 Versteht man unter Makrophänomenen eine eigene gesellschaftliche Ebene sui generis, die nicht auf Mikrosituationen zurückzuführen ist, dann mag eine Mikro-Makro-Dichotomie zutreffend sein. Wenn man diese Sichtweise jedoch ablehnt und mit Schatzki und Latour von einer flat ontology of the social ausgeht (Schatzki 2015, S. 12 ff.; Latour 2005, S. 176), ohne zugleich dem methodologischen Individualismus zu folgen, verändert sich der Blickwinkel deutlich. Dann stehen Makrophänomene nicht mehr ,über“ den Mikrosituationen, sondern sind aus weiteren spezifischen sozialen (räumlich und zeitlich entfernten) Situationen zu rekonstruieren, die auf der gleichen sozialontologischen Ebene angesiedelt sind. Hier ist nicht der Ort, ausführlich auf die Debatte um den Mikro-Makro-Link einzugehen. Wir gehen heuristisch von der Idee der flachen Ontologie aus; von hier aus wäre nach der Konstitution von Momenten der Inter- und Transsituativität zu fragen (zum Überblick vgl. Hirschauer 2014).
} 
fände. Oder noch deutlicher formuliert: Es kann keine Makrokrise geben, die sich nicht auch auf der Ebene von Handlungs- und Interaktionskrisen zeigt.

So notwendig uns eine „mikroskopische Inspektion“ von Handlungs- und Interaktionskrisen erscheint, so wichtig ist es uns auch, die Notwendigkeit zu betonen, die an den Horizonten von Krisensituationen stets sichtbar werdenden gesellschaftlichen bzw. transsituativen Ordnungskonstellationen zur Kenntnis zu nehmen (vgl. KnorrCetina 1981). Welche der die unmittelbare Krisensituation transzendierenden und „rahmenden“" Ordnungen dabei in den Blick geraten, hängt einerseits wesentlich von den theoretischen - um im Bild zu bleiben - Sehinstrumenten und andererseits von der methodischen Perspektive auf den Gegenstand ab. Die Beiträge legen ein vielfältiges Zeugnis davon ab, in welcher Art und Weise Handlungs- und Interaktionskrisen methodisch beobachtbar und erforschbar gemacht werden können. Insofern möchten wir die Leser_innen an dieser Stelle direkt auf die Artikel des Sonderhefts verweisen. Sie reichen methodisch von konzeptuellen Analysen, über Interviewstudien bis hin zu ethnografischen und videointeraktionsanalytischen Ansätzen.

Zuletzt möchten wir darauf hinweisen, dass eine zentrale methodologische Ressource der forschungspraktischen „Nutzung“ von Handlungs- und Interaktionskrisen, seien diese nun fingiert oder ,natürlich“ auftretend, in einem basalen Akt der Befremdung des Unhinterfragten und Unproblematischen besteht. Krisen eröffnen in dieser Hinsicht, auch wenn sie als Gegenstand empirischer Untersuchungen fungieren, nicht nur einen Blick auf die unthematisierten und von den Handelnden oftmals nicht-explizierbaren ,,praktischen Hintergründe“ von Handlungsvollzügen und Interaktionen (etwa in Form impliziten Wissens, eingelebter Routinen oder background expectancies). Sie erlauben es auch, ja machen es sogar notwendig, den soziologisch informierten Blick auf die historisch-kulturell variierende Geltung verschiedener Ordnungsniveaus, Normalitäten und Rationalitäten zu richten (vgl. Adloff et al. 2015). Die Frage nach dem Nicht-Krisenhaften als notwendigem Komplementärbegriff jedweder begrifflichen Fassung von Krisen stellt in diesem Sinne nicht bloß eine Herausforderung für theoretisch-konzeptuelle Analysen dar. Sie zeigt auch insofern immenses soziologisches Potenzial an, als Handlungs- und Interaktionskrisen sowohl den Teilnehmer_innen als auch den soziologischen Beobachter_innen Einsicht in die Relativität und Kontingenz von oftmals als „natürlich“ gegeben erfahrenen Ordnungen gewähren.

Danksagung Wir danken Björn Bosserhoff für das gewissenhafte Lektorieren der Aufsätze und Tilo Grenz für Anregungen zum vorliegenden Text. Die Gutachter_innen haben maßgeblich zum Zustandekommen des vorliegenden Sonderheftes beigetragen. Ihnen sei für ihre kritischen Hinweise und Anregungen herzlich gedankt.

\section{Literatur}

Adloff, Frank, David Kaldewey, und Katharina Gerund (Hrsg.). 2015. Revealing tacit knowledge: Embodiment and explication. Bielefeld: transcript.

Berger, Peter L., und Thomas Luckmann. 2007. Die gesellschaftliche Konstruktion der Wirklichkeit. Eine Theorie der Wissenssoziologie. Frankfurt: Fischer.

Bergmann, Jörg. 2013. Die Trivialität der Katastrophe - Situationen als Grenzobjekte. In Grenzobjekte. Soziale Welten und ihre Übergänge, Hrsg. Reinhard Hörster, Stefan Köngeter, und Burkhard Müller, 285-299. Wiesbaden: Springer VS. 
Blumer, Herbert. 1986. Symbolic Interactionism. Perspective and method. Berkeley: University of California Press.

Dewey, John. 1983. Human nature and conduct. An introduction to social psychology. The middle works. Bd. 14, 1988-1924. Carbondale: Southern Illinois University Press.

Dewey, John. 1998. Die Suche nach Gewißheit. Eine Untersuchung des Verhältnisses von Erkenntnis und Handeln. Frankfurt: Suhrkamp. Übers. Suhr, Martin.

Dewey, John. 2003. Die Elementareinheit des Verhaltens. In Philosophie und Zivilisation, 230-244. Frankfurt: Suhrkamp. Übers. Martin Suhr.

Eberle, Thomas S. 2008. Phänomenologie und Ethnomethodologie. In Phänomenologie und Soziologie. Theoretische Positionen, aktuelle Problemfelder und empirische Umsetzungen, Hrsg. Jürgen Raab, et al., 151-161. Wiesbaden: VS Verlag.

Endreß, Martin, und Nicole Zillien. 2013. Routinen der Krise - Krise der Routinen. Themenpapier zum 37. Kongress der Deutschen Gesellschaft für Soziologie in Trier vom 6. bis 10. Oktober 2014. Soziologie 42:414-422.

Friedrichs, Jürgen. 2007. Gesellschaftliche Krisen. Eine soziologische Analyse. In Die Wahrnehmung von Krisenphänomenen. Fallbeispiele von der Antike bis in die Neuzeit, Hrsg. Helga Scholten, 13-26. Köln: Böhlau.

Garfinkel, Harold. 1967. Studies in Ethnomethodology. Englewood Cliffs: Prentice Hall.

Garz, Detlef, und Uwe Raven. 2015. Theorie der Lebenspraxis. Einführung in das Werk Ulrich Oevermanns. Wiesbaden: Springer VS.

Giddens, Anthony. 1984. The constitution of society. Outline of the theory of structuration. Cambridge: Polity Press.

Goffman, Erving. 1967. Interaction ritual. Essays on face-to-face behavior. New York: Pantheon Books.

Hirschauer, Stefan. 2014. Intersituativität. Teleinteraktionen und Koaktivitäten jenseits von Mikro und Makro. In Interaktion - Organisation - Gesellschaft revisited. Anwendungen, Erweiterungen, Alternativen, Hrsg. Bettina Heintz, und Hartmann Tyrell. Stuttgart: Lucius \& Lucius. Sonderheft der Zeitschrift für Soziologie 45:109-133.

Knorr-Cetina, Karin. 1981. Introduction: The micro-sociological challenge of macro-sociology: Towards a reconstruction of social theory and methodology. In Advances in social theory and methodology. Toward an integration of micro- and macro-sociologies, Hrsg. Karin Knorr-Cetina, und Aaron V. Cicourel, 1-47. Boston: Routledge \& Kegan Paul.

Koselleck, Reinhart. 1982. Krise. In Geschichtliche Grundbegriffe, Bd. 3, Hrsg. Otto Brunner, Werner Conze, und Reinhart Koselleck, 617-650. Stuttgart: Klett-Cotta.

Latour, Bruno. 2005. Reassembling the social. An introduction to Actor-Network-Theory. Oxford: Oxford University Press.

Luhmann, Niklas. 2007. Öffentliche Meinung. In Politische Planung. Aufsätze zur Soziologie von Politik und Verwaltung, 9-35. Wiesbaden: VS Verlag.

Mead, George Herbert. 1973. Geist, Identität und Gesellschaft aus der Sicht des Sozialbehaviorismus. Frankfurt: Suhrkamp. Übers. Ulf Pacher.

Mead, George Herbert. 1987. Die Definition des Psychischen. In Gesammelte Aufsätze, Bd. 1, 83-148. Frankfurt: Suhrkamp. Übers. Klaus Laermann et al.

Merleau-Ponty, Maurice. 1966. Phänomenologie der Wahrnehmung. Berlin: De Gruyter. Übers. Rudolf Boehm.

Oevermann, Ulrich. 2006. Wissen, Glauben, Überzeugung. Ein Vorschlag zu einer Theorie des Wissens aus krisentheoretischer Perspektive. In Neue Perspektiven der Wissenssoziologie, Hrsg. Dirk Tänzler, Hubert Knoblauch, und Hans-Georg Soeffner, 79-118. Konstanz: UVK.

Oevermann, Ulrich. 2008. „Krise und Routine“ als analytisches Paradigma in den Sozialwissenschaften. Abschiedsvorlesung. https://www.agoh.de/lit/index.php?action=resource_RESOURCEVIEW_ CORE\&id=1686. Zugegriffen: 19. März 2016.

Plessner, Helmuth. 2003. Lachen und Weinen. Eine Untersuchung der Grenzen menschlichen Verhaltens. In Ausdruck und menschliche Natur. Gesammelte Schriften, Bd. VII, 201-387. Frankfurt: Suhrkamp.

Schatzki, Theodore. 2015. Spaces of practices and of large social phenomena. http://www.espacestemps. net/en/articles/spaces-of-practices-and-of-large-social-phenomena/. Zugegriffen: 18. März 2016.

Schubert, Cornelius, und Werner Rammert. 2006. Unsicherheit und Mehrdeutigkeit im Operationssaal. Routinen und Risiken verteilter Aktivitäten in Hightech-Arbeitssituationen. In Technografie. Zur Mikrosoziologie der Technik, Hrsg. Werner Rammert, und Cornelius Schubert, 313-339. Frankfurt: Campus. 
Schütz, Alfred. 2003. Strukturen der Lebenswelt. In Theorie der Lebenswelt 1. Die pragmatische Schichtung der Lebenswelt. Alfred Schütz-Werkausgabe, Bd. V, Hrsg. Martin Endreß, und Ilja Srubar, 325-347. Konstanz: UVK.

Schütz, Alfred. 2004. Das Wählen zwischen Handlungsentwürfen. In Relevanz und Handeln 1. Zur Phänomenologie des Alltagswissens. Alfred Schütz-Werkausgabe, Bd. VI.1, Hrsg. Elisabeth List, 251-300. Konstanz: UVK.

Sebald, Gerd. 2014. Generalisierung und Sinn. Überlegungen zur Formierung sozialer Gedächtnisse und des Sozialen. Konstanz: UVK.

Alexander Antony M.A., ist wissenschaftlicher Mitarbeiter am Institut für Soziologie der Universität Wien. Forschungsschwerpunkte: Handlungs- und Praxistheorien, Wissenssoziologie, Körper- und Emotionssoziologie, Methodologie und Methoden interpretativer Sozialforschung. Ausgewählte Veröffentlichung: Tacit Knowledge and Analytic Autoethnography. Methodological Reflections on the Sociological Translation of Self-Experience. In: Revealing Tacit Knowledge. Embodiment and Explication, Hrsg. Frank Adloff, Katharina Gerund und David Kaldewey, 139-167. Bielefeld: transcript 2015.

Gerd Sebald PD Dr., ist Koordinator am Graduiertenkolleg 1718 „Präsenz und implizites Wissen“ an der FAU Erlangen-Nürnberg. Seine Forschungsschwerpunkte sind soziologische Theorie, Wissens-, Kulturund Mediensoziologie, soziale Gedächtnisse und die Geschichte der Soziologie. Ausgewählte Veröffentlichung: Generalisierung und Sinn. Überlegungen zur Formierung sozialer Gedächtnisse und des Sozialen. Konstanz: UVK 2014.

Frank Adloff Prof. Dr., hat den Lehrstuhl für Allgemeine und Kultursoziologie an der FAU ErlangenNürnberg inne. Seine Forschungsschwerpunkte liegen in den Bereichen soziologische Theorie, Zivilgesellschaft, Theorie der Gabe und Konvivialität, Kultursoziologie und Emotionssoziologie. Ausgewählte Veröffentlichungen: Gewohnheiten, Affekte und Reflexivität. Ein pragmatistisches Modell sozialer Kooperation im Anschluss an Dewey und Mead. In: Potentiale einer pragmatistischen Sozialtheorie. Beiträge anlässlich des 150. Geburtstags von George Herbert Mead, Hrsg. Frithjof Nungesser und Franz Ofner. Sonderheft der Österreichischen Zeitschrift für Soziologie 2013, 12:21-41 (mit Dirk Jörke); Gifts of Cooperation, Mauss and Pragmatism. London: Routledge 2016. 